\title{
Effect of Anderson Localization on Auger Destruction of DNA
}

\author{
N. N. Turaeva, ${ }^{1}$ S. Schroeder, ${ }^{1}$ and B. L. Oksengendler ${ }^{2}$ \\ ${ }^{1}$ Biological Department, Webster University, 470 East Lockwood, Webster Groves, MO 63119, USA \\ ${ }^{2}$ Theoretical Department, Institute of Polymer Chemistry and Physics, Kadiri 7B, 100128 Tashkent, Uzbekistan
}

Correspondence should be addressed to S. Schroeder, schroeds@webster.edu

Received 5 November 2012; Accepted 21 November 2012

Academic Editors: K. Nishizawa and M. P. Ponomarenko

Copyright ( $) 2012$ N. N. Turaeva et al. This is an open access article distributed under the Creative Commons Attribution License, which permits unrestricted use, distribution, and reproduction in any medium, provided the original work is properly cited.

\begin{abstract}
The effect of Anderson localization in DNA on the Auger destruction by the Coulombic explosion at ionized radiation has been theoretically discussed in the present work. The theory of Auger destruction of DNA has been modified taking into account the localized and delocalized electron states in DNA owing to the correlated disorder in a sequence of nucleotides. According to the modified theoretical model of Auger destruction, the dominant ratio of delocalized states to localized states in exon compared to intron results in stronger radiation resistance of exons to ionized irradiation causing the Auger-cascade process than the radiation resistance of introns.
\end{abstract}

One of the interesting and important applications of the localization theory developed for one-dimensional systems [1-4] is related to conductivity of DNA molecules [4-6]. The electronic structure of DNA represents a very interesting problem by itself. Surely the mobility of electrons along the sequence effects DNA's functional behavior (information transferring, enzyme-DNA interactions, etc.) and its structural-sequential integrity. The charge migration is believed to be important for the radiation damage repair [7]. The recent study has shown that single-base mutations on DNA molecules may lead to significant changes in conductance [8]. Since most of the mutations in DNA are successfully healed, they assumed the existence of charge transport through delocalized states, responsible for the transfer of information at much longer distances.

DNA macromolecule is considered to be a long sequence of discrete site potentials-basic nucleotides. The electron transport along this sequence occurs due to hopping between the neighboring nucleotides. This property justifies the application of the tight binding model. An uncorrelated sequence of nucleotides localizes all quantum electron states, as occurs in one-dimensional white-noise potential, making impossible the charge transfer at distances longer than the localization length. Studies of long-range correlation in nucleotide structure resulted in proclamation that DNA was aperiodic crystal with long-range correlation [9]. In the work [4-6] in the framework of the two-channel tight-binding model it was shown how the information coded in a sequence of nucleotides may affect the localization length (therefore, the resistivity) of a given segment of a DNA molecule. The principal results of these work are that for the most of the energies the localization length inside the exon (the coding regions of DNA) region exceeds by order of magnitude the localization length inside the intron region (the noncoding regions of DNA). This confirms the fact that very different kinds of information are coded in these regions. The presence of extended states in the spectrum of exons regions strongly supports a hypothesis that the information about DNA repair processes transfers in a damaged DNA along a nucleotide sequence due to electrical mechanisms. Since proteins detect the damaged region in a sequence by virtue of transport properties [10-12], it is natural that the coding regions (exons) have extended states, while this property is irrelevant for the noncoding regions (introns) [5]. One of the factors causing the point mutation in DNA is ionized radiation $[13,14]$, which was able to ionize atoms and to break chemical bonds and disrupt the large molecules. At direct and indirect radiation influence of ionized radiation on DNA can cause one-strand break and two-strand breaks of DNA, biological response to which will be gene mutation, cell death, and others. In the work [7] the effect of charge transfer on oxidative thymine dimer repair in the DNA helix at UV radiation was considered. They established that repair efficiency did not decrease with increasing distance 
between intercalated rhodium noncovalently bonded to the DNA duplex and the thymine dimer, but it diminished with disruption of the intervening $\pi$-stack.

In this work we consider the dependence of bond break probability upon the electron localization lengths differing in coding and noncoding sequences at realization of Coulombic explosion at direct ionized irradiation of DNA.

It well known that if DNA is exposed to ionized irradiation as X-ray, gamma, and UV radiation the bond break in DNA can occur by the mechanism of Coulombic explosion [15-17]. According to [17] the mechanism of Coulombic explosion includes three consecutive processes: (1) the ionization of multielectron atoms of DNA (e.g., phosphorous, oxygen atoms) with cross-section of $\sigma_{i},(2)$ the Auger-cascade process, in result of which multicharged $(Z)$ ions are created with the probability of $\alpha_{i}(Z)$, and (3) the decay of nonstable ionic state by ions displacement accompanied by the recovery process of electrons filling up the positive charged ions with the probability of $W\left(\tau_{+} / \tau_{e}\right)$. Here $\tau_{+}$is the time of gaining the kinetic energy to multicharged ions flying apart from their sites enough for overcoming the bond energy (about $5 * 10^{-14} \mathrm{~s}$ ); $1 / \tau_{e}$ is the probability of electrons filling up the multipositively charged ionic state (for semiconductor crystals about $10^{-16} \mathrm{~s}$ ).

The value of $\sigma_{i}$ depends on the type of radiation (photons, electrons, and ions) and the deep shell electron energy. The probability of $\alpha_{i}(Z)$ depends upon the wave functions of electrons involved into the Auger cascade. The total cross-section of Auger destruction of molecule is defined by the multiplication of probabilities of these independent successive processes as

$$
\sigma_{A-d}=\sigma_{i} \alpha_{i}(Z) W\left(\frac{\tau_{+}}{\tau_{e}}\right) .
$$

We can expect the electron localization effect on the third process which is very important because of the destruction DNA can occur in spite of electrons neutralization of localized positive charges. The probability of this process can be defined by the following expression [17]:

$$
W=\exp \left(-\frac{\tau_{+}}{\tau_{e}}\right)
$$

The value of $1 / \tau_{e}$ is associated with the $Z$-positively charged ions relaxation by localized $\left(1 / \tau_{e}^{\text {loc }}\right)$ and delocalized $\left(1 / \tau_{e}^{\text {deloc }}\right)$ electron states in the following way:

$$
\frac{1}{\tau_{e}}=\beta_{\mathrm{loc}} \frac{1}{\tau_{e}^{\text {loc }}}+\beta_{\mathrm{deloc}} \frac{1}{\tau_{e}^{\text {deloc }}}
$$

Here $\beta_{\text {loc }}$ and $\beta_{\text {deloc }}$ are the fractions of localized and delocalized electron states, which are different for exon and intron parts of DNA. According to the references [4-6] the fraction of delocalized states for exon is greater than for intron. Since the spatial extensions of electron localized and delocalized states are different, we can expect that the probability of filling the positively multicharged states up by delocalized electrons is greater than by localized electrons, that is:

$$
\frac{1}{\tau_{e}^{\text {loc }}} \ll \frac{1}{\tau_{e}^{\text {deloc }}}
$$

The total cross-section of Auger destruction of molecule can be defined taking into account the expression (3) in the following way:

$$
\sigma_{A-d}=\sigma_{i} \alpha_{i}(Z) \exp \left[-\tau_{+}\left(\beta_{\mathrm{loc}} \frac{1}{\tau_{e}^{\mathrm{loc}}}+\beta_{\text {deloc }} \frac{1}{\tau_{e}^{\text {deloc }}}\right)\right] .
$$

Based on the dominant ratio of delocalized states related to localized ones for exons rather than introns [4-6] and the expression (4) we can conclude that the electron recovery of positive multicharged states resulting from Auger-cascade process at ionized radiation is more efficient in the coding sequence of DNA than in the noncoding sequence. It means the cross-section of Auger destruction will be less for exons than for introns according to the expression (5). We can assume that the stronger radiation resistance of exons compared to introns in DNA may be crucial for survival of genetic information during longlife evolution on the strong irradiation background.

\section{References}

[1] P. W. Anderson, "Absence of diffusion in certain random lattices," Physical Review, vol. 109, no. 5, pp. 1492-1505, 1958.

[2] N. F. Mott and W. D. Twose, "The theory of impurity conduction," Advances in Physics, vol. 10, no. 38, pp. 107-163, 1961.

[3] D. J. Thouless, "Electrons in disordered systems and the theory of localization," Physics Reports, vol. 13, no. 3, pp. 93-142, 1974.

[4] A. A. Krokhin, V. M. K. Bagci, F. M. Izrailev, O. V. Usatenko, and V. A. Yampol'Skii, "Inhomogeneous DNA: conducting exons and insulating introns," Physical Review B, vol. 80, no. 8, Article ID 085420, 6 pages, 2009.

[5] C. T. Shin, "Characteristic length scale of electric transport properties of genomes," Physical Review E, vol. 74, no. 1, Article ID 010903(R), 4 pages, 2006.

[6] K. Bagci, Anderson localization in two-channel wires with correlated disorder: DNA as an application [Dissertation], University of North Texas, 2007.

[7] P. J. Dandliker, R. E. Holmlin, and J. K. Barton, "Oxidative thymine dimer repair in the DNA helix," Science, vol. 275, no. 5305, pp. 1465-1468, 1997.

[8] J. Hihath, B. Xu, P. Zhang, and N. Tao, "Study of singlenucleotide polymorphisms by means of electrical conductance measurements," Proceedings of the National Academy of Sciences of the United States of America, vol. 102, no. 47, pp. 16979-16983, 2005.

[9] C. K. Peng, S. V. Buldyrev, A. L. Goldberger et al., "Longrange correlations in nucleotide sequences," Nature, vol. 356, no. 6365, pp. 168-170, 1992.

[10] S. R. Rajski, B. A. Jackson, and J. K. Barton, "DNA repair: models for damage and mismatch recognition," Mutation Research, vol. 447, no. 1, pp. 49-72, 2000.

[11] E. Yavin, A. K. Boal, E. D. A. Stemp et al., "Protein-DNA charge transport: redox activation of a DNA repair protein by guanine radical," Proceedings of the National Academy of Sciences of the United States of America, vol. 102, no. 10, pp. 3546-3551, 2005.

[12] T. Hu and B. I. Shklovskii, "How does a protein search for the specific site on DNA: the role of disorder," Physical Review E, vol. 74, no. 2, Article ID 021903, 4 pages, 2006.

[13] W. Han and K. N. Yu, "V. 4.," in Advances in Genetics Research, K. V. Urbano, Ed., Nova Science, New York, NY, USA, 2010. 
[14] L. E. Feinendegen, "Biological damage from the Auger effect, possible benefits," Radiation and Environmental Biophysics, vol. 12, no. 2, pp. 85-99, 1975.

[15] K. G. Hofer, "Biophysical aspects of Auger processes: a review," Acta Oncologica, vol. 35, no. 7, pp. 189-196, 1996.

[16] E. Parilis, L. Kishinevskiy, N. Turaev et al., Atomic Collisions on Solid Surfaces, Elsevier Science Publisher BV, New York, NY, USA, 1993.

[17] B. L. Oksengendler, N. N. Turaeva, I. N. Ruban, and S. S. Rashidova, "Auger destruction of polymers," Doklady Chemistry, vol. 387, no. 1-3, pp. 302-303, 2002. 

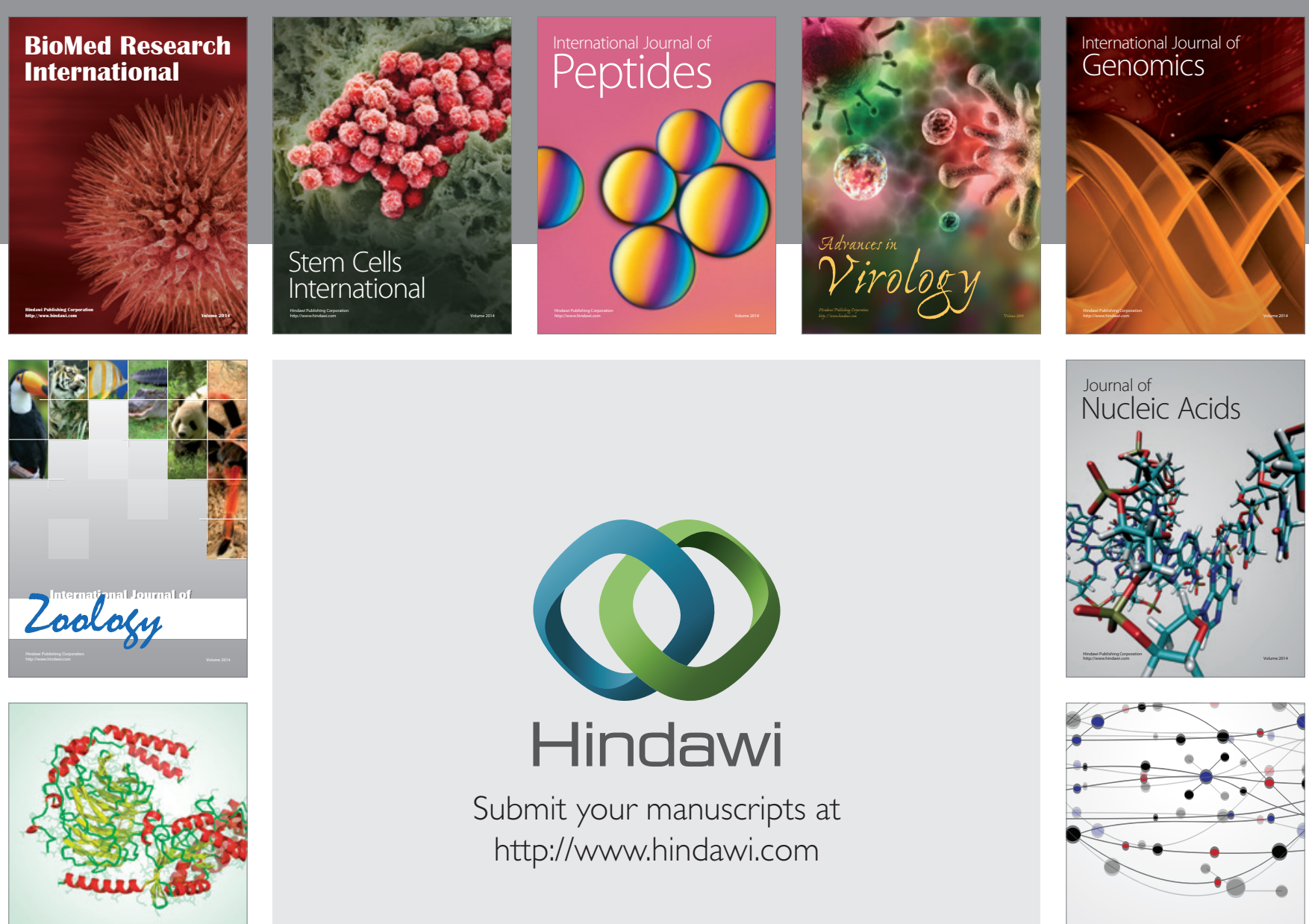

Submit your manuscripts at

http://www.hindawi.com

Signal ${ }^{\text {Jumal }}$ Transduction
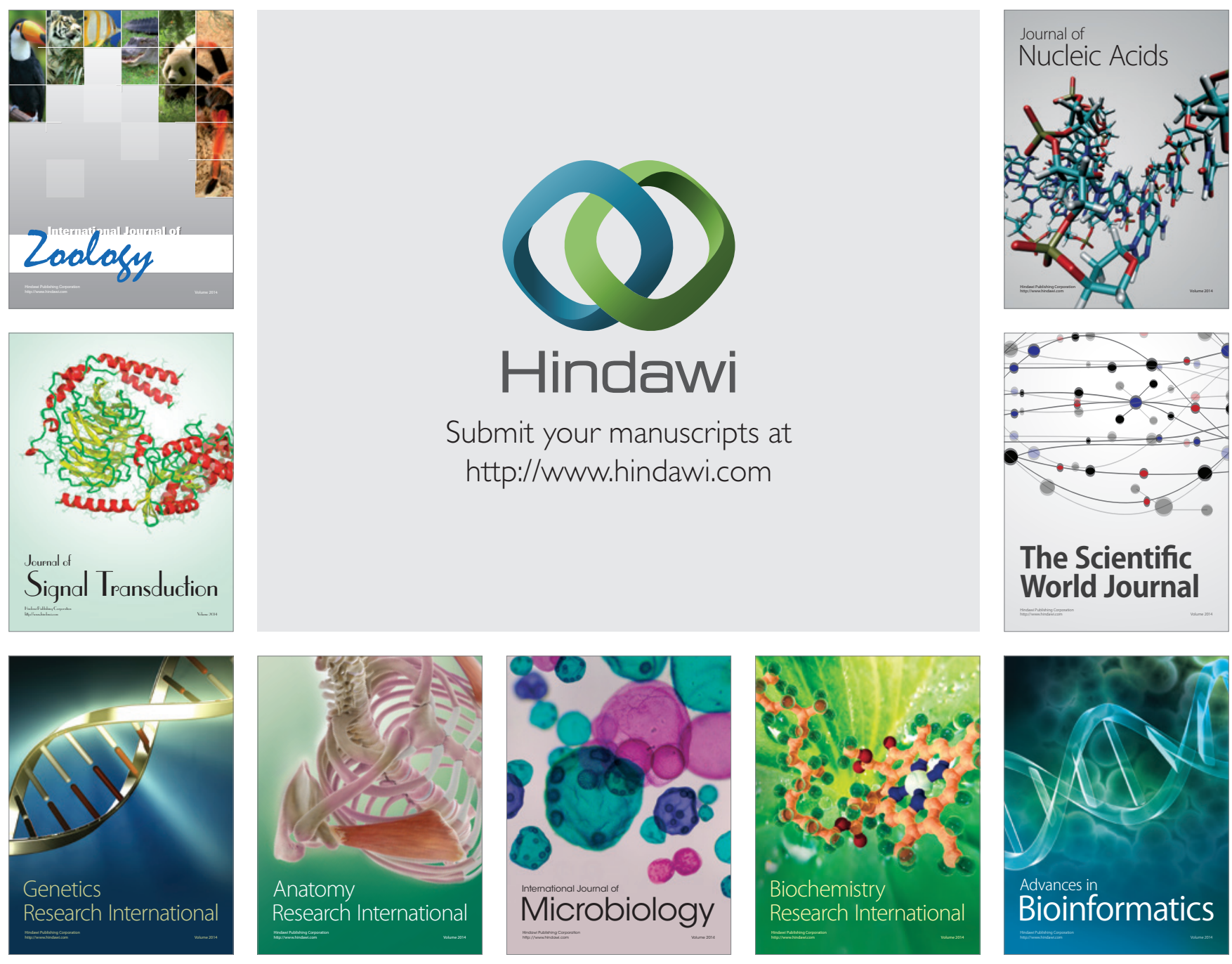

The Scientific World Journal
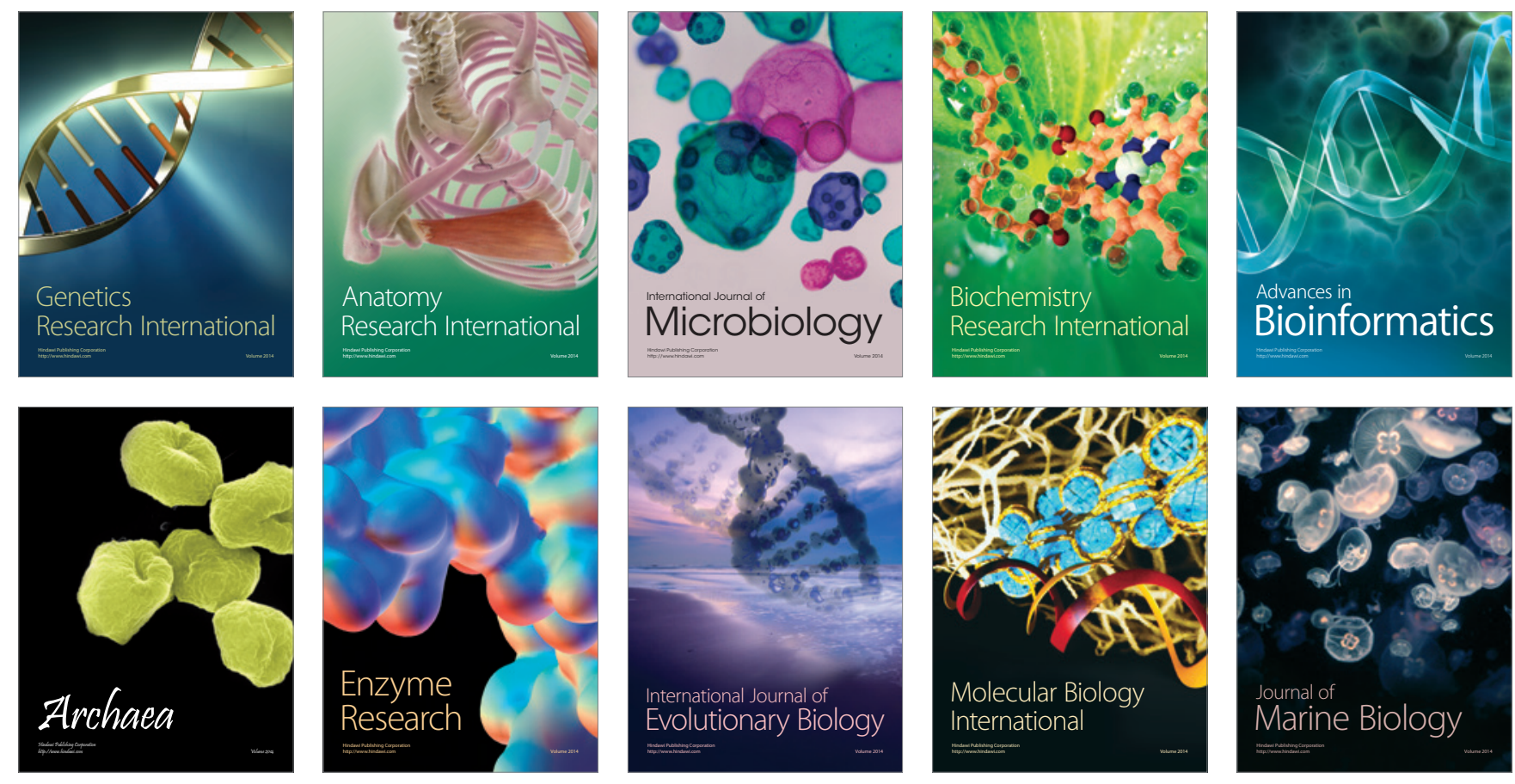